\title{
Information in Repeated Ultimatum Game with Unknown Pie Size
}

\author{
Ching Chyi Lee and William K. Lau \\ Department of Decision Sciences and Managerial Economics, Faculty of Business Administration, \\ The Chinese University of Hong Kong, Hong Kong
}

Correspondence should be addressed to William K. Lau; williamlau@cuhk.edu.hk

Received 24 August 2012; Revised 15 November 2012; Accepted 27 November 2012

Academic Editor: Philip J. Grossman

Copyright (C) 2013 C. C. Lee and W. K. Lau. This is an open access article distributed under the Creative Commons Attribution License, which permits unrestricted use, distribution, and reproduction in any medium, provided the original work is properly cited.

\begin{abstract}
Within existing literature, it is well known that people's behavior in ultimatum game experiments cannot be explained by perfect rationality model. There is, however, evidence showing that people are boundedly rational. In this paper, we studied repeated ultimatum game experiments in which the pie size is only known to the proposer (player 1), but the transaction history is made known to both players. We found that subject's behavior can be very well explained by the history-consistent-rationality model (HCR model) of Lee and Ferguson (2010), which suggests that people's behavior is affected by what they observed in the past. The HCR model is able to yield point predictions whose errors are on average within $5 \%$ of the total pie size. The experimental results provide evidence that subjects' behavior is boundedly rational with respect to the transaction history.
\end{abstract}

\section{Introduction}

Within the existing ultimatum game literature, it is widely held by economists that game theory fails to predict the subjects' behaviors accurately. Implicit in this evidence are the conjecture of altruistic concerns and the matter of fairness (see, e.g., [1, 2]). While it is commonly known that the decision of accepting or rejecting an offer in ultimatum games depends on respondent's tolerance of unfairness, there have been no prescriptive models in the literature for suggesting the optimal offer that proposer should propose. In this paper, we demonstrate that the history-consistent rationality (hereafter, HCR) model can give point prediction to the proposer's offer in the ultimatum game. This kind of quantitative prediction is different from the past literature which focuses on qualitative prediction.

Our research contributions are of twofold. First, our experimental design simulates the real market condition to allow us to better understand how the real economy works. In the existing literatures, scholars have studied ultimatum games with asymmetric information to approximate the real life bargaining situation, as people often do not know how much there is at stake for the other person [3-5]. In our research, we replicate the real market condition by further allowing market information to be available to every subject in the experiment. Consider a person purchasing a house, he would certainly collect market information to bargain for a better deal, because the reservation price of the house owner is usually unknown to the buyer. In each session of our experiments, there are eighteen to twenty pairs of subjects, playing repeated ultimatum games up to twenty periods. The market information for all accepted and rejected offers in the previous periods is made available to all subjects, including both the proposers and respondents. We ran four sessions of experiments with two different pie sizes to approximate the low and high real estate markets.

Secondly, we propose a prescriptive model that suggests the optimal strategies players should adopt and found that players do, to a large extent, follow our prescriptive model. Raiffa [6] conjectured that subjects in economic games do not consider their opponents as perfectly rational game players, but rather formulating their strategies as if they are making decision under uncertainty. We follow his track and assume that the proposers in the ultimatum game treat each respondent's cutoff price for acceptance as a random variable governed by a cumulative probability function $F(x)$. 
Therefore, proposers should choose an offer $x$ to maximize his expected payoff, $x F(x)$. Our experimental design provides sufficient conditions to test our conjecture as (1) the game is played repeatedly with different opponents and (2) the historical market information is made available to the players, which allows proposers to construct $F(x)$ from historical data and make the optimal offer that maximize $x F(x)$. If it is without repetition, market information will be irrelevant and estimate of $F(x)$ cannot be formed. Furthermore, because the game is played with unknown pie size, so offers will not converge to 50:50 split too quickly, thus providing enough data points for us to test our model.

We found that the history-consistent rationality model (HCR model) suggested by Lee and Ferguson [7] can explain the subjects behavioral quite well. On average, the prediction error made by the HCR model is within 5\% of the total pie size. The HCR model is robust in predicting the subjects' behaviors with different pie sizes.

The organization of this paper is as follows. Section 2 provides a literature review for the ultimatum game experiments. Section 3 discusses the research design and methodology. In Section 4, a history-consistent rationality model is adopted to predict and explain subject behaviors across periods in a repeated ultimatum game. Section 5 summarizes the major findings and concludes this paper.

\section{Literature Review}

The ultimatum game has been an area of extensive studies. In ultimatum game, strategic interaction occurs only in the form of anticipating future decisions. Bargaining processes are often modeled as ultimatum bargaining games [8], because only when knowing what drives the individual decisions in simple games, one can be sure how to interpret the results of more complex situations. It is well known in the economic literature that subjects do not anticipate future decisions in the way which characterizes the individually rational decision behavior in ultimatum games. Güth et al. [9] ran the first ultimatum game in the economic literature and found that instead of proposing a minimal amount as suggested by game theory, the modal proposal is a 50:50 split, with the mean demand of proposers being $65 \%$ of the total pie, leaving $35 \%$ to respondents (Güth et al. [9] ran two ultimatum game sessions-one with naive and another with experienced players. The mean demand of $65 \%$ share is the result of naive players.). Similar results were obtained from the ultimatum game experiments conducted in the later years (see $[10,11])$.

Ochs and Roth [12] showed that the results of the ultimatum game provide nearly no support for the descriptive value of game theory, as the theoretical mean offer always lies outside two standard deviations of the actual mean offer on any trials. Scholars continuously found evidences that subjects are not perfectly rational, as they do not make decision purely based on monetary incentives: respondent always rejects positive offers when the offers are notably less than what proposer kept for himself. For a comprehensive review of the relevant experiments conducted, see Camerer and Thaler [11] and Thaler [13]. To explain why respondents reject nonzero offers, Bolton [14], Fehr and Schmidt [2], and Trautmann [15] have proposed different descriptive models.

Bolton [14] considered bargainers behave as if they are negotiating both absolute and relative money and proposed a model suggesting that players have a utility function $U\left(x_{r}, x_{r} / x_{p}\right)$, where $x_{r}$ is the absolute amount respondents got, and $x_{r} / x_{p}$ is the ratio of respondent's share to proposer's share. This form of the utility function suggests that the respondent cares not only how much he/she gets, but also how much the proposal gets, giving the rationale for the respondent's rejecting unfair offer. Fehr and Schmidt [2] shared similar ideas and built a more comprehensive mathematical model to calculate player $i$ 's utility with consideration on others' payoff as follows:

$$
\begin{aligned}
U_{i}(x)= & x_{i}-\alpha_{i} \frac{1}{n-1} \sum_{j \neq i} \max \left\{x_{j}-x_{i}, 0\right\} \\
& -\beta_{i} \frac{1}{n-1} \sum_{j \neq i} \max \left\{x_{i}-x_{j}, 0\right\},
\end{aligned}
$$

where $x_{i}$ is the payoff to player $i, n$ is the number of players, $\beta_{i} \leq \alpha_{i}$ and $0 \leq \beta_{i} \leq 1$; coefficients $\alpha$ and $\beta$ represent the weight of envy and guilt.

When coefficients $\alpha$ and $\beta$ are zero, players are purely self-interested, so the standard perfect rationality assumption of players is a special case of this model. Similar to Bolton's model, this model also suggests that respondents in ultimatum games may reject low offers to enforce equality, and it is a dominant strategy for respondents to accept any offers $x \geq 0.5$, and to reject $x$ if

$$
x<\frac{\alpha_{2}}{1+2 \alpha_{2}}
$$

where $\alpha_{2}$ is the weight of envy of respondent, and the pie size is normalized to 1 .

Proposers, believing $\alpha_{2}$ is distributed with a cumulative distribution function $F\left(\alpha_{2}\right)$, where $F\left(\alpha_{2}\right)$ has supported $[\underline{\alpha}, \bar{\alpha}]$ with $0 \leq \underline{\alpha}<\bar{\alpha}<\infty$, have the optimal strategy of proposing the following:

$$
x^{*} \begin{cases}=0.5, & \text { if } \beta_{i}>0.5, \\ \in\left[\frac{\bar{\alpha}}{1+2 \bar{\alpha}}, 0.5\right], & \text { if } \beta_{i}=0.5, \\ \in\left[\frac{\underline{\alpha}}{1+2 \underline{\alpha}}, \frac{\bar{\alpha}}{1+2 \bar{\alpha}}\right], & \text { if } \beta_{i}<0.5,\end{cases}
$$

where $\beta_{1}$ is the weight of guilt of proposer.

Though Fehr-Schmidt model successfully explains subject behaviors in a qualitative way, it could not address the issue that respondents aim at "punishing unfairness, not rejecting inequality" in ultimatum games; that is, they could accept a computer generated low offer more readily than a human decided low offer. Hence, Trautmann [15] modified their model to incorporate process fairness and successfully testified the new model by a random ultimatum game, which uses a random device to propose an allocation of the pie to 
the proposer and respondent. The modified model for the two-person ultimatum game is

$$
\begin{aligned}
U_{i}\left(x_{i}, X, Y\right)= & x_{i}-\alpha_{i} \max \{E[Y]-E[X], 0\} \\
& -\beta_{i} \max \{E[X]-E[Y], 0\},
\end{aligned}
$$

where $x_{i}$ is the payoff to the player $i, \beta_{i} \leq \alpha_{i}$ and $0 \leq \beta_{i} \leq 1$; coefficients $\alpha$ and $\beta$ represent the weight of envy and guilt; $E[X]$ is the expected payoff to the player $i$, and $E[Y]$ is the expected payoff to the other player.

Trautmann's model captures the expected values rather than the actual outcomes in the game and focuses on the process fairness rather than the outcome fairness. However, since it is technically impossible to measure each subject's $\alpha$ and $\beta$, all these proposed models are essentially qualitative in nature-they cannot yield a numerical prediction of what proposers should offer to responders.

Güth [16], after actively involved in ultimatum bargaining experiments for many years, concluded that human decision making is a dynamic reasoning process guided by past experiences and limited strategic considerations. Thinking along the same line, we conjectured that subjects tend to choose the previously successful mode of behavior and formulate their strategies accordingly. We modeled the optimal subject behaviors with the history-consistent rationality model, which assumes players respond to each other "rationally," but their rationality is bounded by their knowledge about the game and how others play, and their knowledge is derived from historical data. In the following sections, we will show how this model could explain the actual human behaviors, supported by experimental data.

\section{Experimental Design}

The ultimatum game is a stylized bargaining situation that has been used to examine a broad range of behaviors [11]. In its simplest form, proposer proposes an allocation of a fixed sum of money, and the respondent chooses either to accept or reject the proposal. If the proposal is accepted, the money will be distributed accordingly; otherwise, both players receive nothing.

In our experiments, we simulate the real estate market condition by allowing subjects to access the historical market information in repeated ultimatum games with unknown pie size. Our experimental design provides sufficient conditions to test the HCR model, which argues that people make decision based on historical information. In our experiment, the game is played repeatedly with different opponents and the historical market information is made available to the players, which allows proposers to construct a belief $F\left(p_{t}\right)$ in each period from the historical data about the probability that the offer $p_{t}$ will be accepted by the respondent. This belief is then used to determine the optimal offer that maximize the respondent's expected payoff $p_{t} F\left(p_{t}\right)$. If it is without repetition, market information will be irrelevant and estimate of $F\left(p_{t}\right)$ cannot be formed. Furthermore, because the game is played with unknown pie size, offers will not converge to $50: 50$ split too quickly, thus providing enough data points for us to test our model.
Four experimental sessions with two different pie sizes, HK\$136 ( US\$17) and HK\$217 ( US\$28), were conducted. We intentionally made the pie sizes difficult to be guessed by respondents to avoid offers being converged too quickly, so that we could gather sufficient data to test our proposed model. Forty and thirty-six business students participated in two separated sessions of $\mathrm{HK} \$ 136$; forty and thirty-six business students participated in two separated sessions of HK\$217, respectively. Each session lasted about 60 minutes. Subjects were randomly assigned to the role of proposer or respondent at the beginning of the experimental session, and they played the same role for the entire session. In each game, however, they were matched with a different, anonymous opponent. The entire session consisted of 20 periods, and the number of games of play was unknown to the subjects. In each game, the randomly paired subjects are asked to distribute a fixed amount, which is the private information to the proposer. After the proposer makes a proposal, the respondent has to decide whether to accept or reject the proposal without knowing the total pie size. The common knowledge that both proposers and respondents possess is the game history, that is, the accepted offers and rejected offers in the market during the previous rounds.

Prior to the start of the play, self-paced instructions were presented via individual Power Point presentations that included interactive questions to assess understanding of the game (the game instruction is shown in Supplementary Material available online at http://dx.doi.org/ 10.1155/2013/470412 (see Appendix 1)). Experiments were conducted via computers in a laboratory arranged so that it is impossible for participants to know the identity of the other negotiators or to see others' screens. The proposers' proposed amount and the decision of acceptance or rejection by respondents are all transmitted via the terminals. No other communications among players are allowed. Each screen also displays a complete market history of accepted offers and rejected offers. To motivate participants, they are informed that they would be paid the average of their net payoff from five randomly selected periods. For the session with HK\$136 pie size, $\mathrm{HK} \$ 20$ is given to each subject as seed money at the beginning of the experiment. On average, participants earned $\mathrm{HK} \$ 84$ for a session (the amount of potential winning is very attractive to the undergraduate participants in order to motivate them to play the game seriously. For comparison, the hourly rate for an on-campus job is $\mathrm{HK} \$ 50)$.

\section{A Predictive Model for Ultimatum Game}

All models with perfect rationality assumption, such as perfect Bayesian equilibrium, predict the proposer should propose a minimum amount to the respondent, as a rational respondent will accept any amounts that are greater than zero.

Models without perfect rationality assumption can be divided into two types. The first type is qualitative in nature, such as Bolton [14], Fehr and Schmidt [2], and Trautmann [15], which contains variables that are neither measurable nor observable by players in the ultimatum game. Thus, these models could only give a directional prediction. For 
instance, it is not possible for players to observe the value of the Fehr-Schmidt model's $\alpha$ and $\beta$ of each player in the ultimatum game. Furthermore, our data show that the offers are increasing across periods, which indicates that either the proposers are updating their believes about the distribution of respondents' envy component across periods, or the values of $\alpha$ and $\beta$ are simply not fixed for individuals as suggested by Fehr and Schmidt.

Another type of models without perfect rationality assumption is quantitative in nature, but most of these models also predict the proposers should offer a minimal amount. For instance, Camerer et al. [17] proposed a renowned cognitive hierarchy model, which relaxes the perfect rationality assumption and suggests that players only do $k$ steps of the iterative thinking process, and assume their opponents are distributed, according to a normalized Poisson distribution, from step 0 (who simply randomized their choice) to step $k-1$. Apparently, this model predicts proposers either randomize their offers (if $k=0$ ) or will propose a minimal amount when $k \geq 1$.

In reality, researchers found proposers typically offer $30 \%$ to $40 \%$ on average, with a $50-50$ split often the mode in the ultimatum games. Those offers less than $20 \%$ of the pie size are frequently rejected [11]. To account for this phenomenon, we analyzed four repeated ultimatum game experiments with unknown pie size to the respondents, and adopt the HCR model proposed by Lee and Ferguson [7], which is a bounded rationality model derived from the concept of fictitious play, to calculate proposer's optimal offer.

The HCR model assumes players respond to each other "rationally," but their rationality is bounded by their knowledge about the game and how others play, and their knowledge is derived from historical data. In other words, players behave rationally given the information learned from history. We conjecture that there are two types of possible rationalities for players to best response to the historical distribution of play in the market, namely herd behavior and fictitious play.

Herd Behavior. Banerjee [18] argued that it is rational for a decision maker to look at the decisions made by previous decision makers in taking him/her own decision, because these other decision makers may have some information that is important to him/her. Banerjee showed that the decision rules chosen by optimizing individuals could be characterized by herd behavior; that is, people would follow what others were doing rather than using their information.

Following Banerjee's argument, we define proposers who follow herd behavior would choose the "mode" of the accepted offers in the previous two periods as their proposed offers to respondents, as most of the subjects indicated, in an ex-post experiment survey, (see Supplementary Material (Appendix 2)), that they used the historical market information in the previous two rounds to assist them in making decision.

Fictitious Play. Brown [19] coined the term fictitious play, which suggested that players in every round play a myopic pure best response against the empirical strategy distribution of their opponents. Following Brown's logic, we define proposers who follow fictitious play would maximize their expected payoff based on the historical distribution of play, so the proposer's offer could be predicted as follows.

Based on history, for each game the proposer forms a belief about the probability, $F\left(p_{t}\right)$, that an offer $p_{t}$ will be accepted. The proposer then chooses an offer $p_{t}$ to maximize his expected payoff, that is, $p_{t} F\left(p_{t}\right)$. Although proposers may form beliefs in different way, intuitively, each proposer's belief $F\left(p_{t}\right)$ can be estimated by fitting a logistic regression model. For game $t$, the dependent variable $A_{t}$ of the logistic model is defined as follows:

$$
A_{t}= \begin{cases}1, & \text { if the proposer's offer was accepted } \\ 0, & \text { otherwise. }\end{cases}
$$

As the processing capacity of human beings is limited, we can assume that the proposers will only use previous $x$ periods (i.e., from $t-x-1$ to $t-1$ periods) to estimate the value of $F\left(p_{t}\right)$. A questionnaire (see Supplementary Material (Appendix 2)) is distributed to each subject after the experiment to ask them how many previous periods of market information (if any) they used for making decision. Most of the subjects indicated that they only used the previous two periods. Hence, we use two-period historical data to predict the optimal subject behaviors.

The HCR model is a prescriptive model that could quantify the optimal strategies that players should adopt. The experimental result illustrates that the HCR model successfully yields an accurate point prediction for the proposer's offer that is on average within $5 \%$ absolute deviation of the total pie size. Four ultimatum experiments with different pie sizes were tested, and the HCR model is robust in different experimental conditions.

4.1. Description of Experiment Results. The detailed distribution of offers across periods in each session is shown in the box-and-whisker plots in Supplementary Material (see Appendix 3). We found that the accepted offers converge to around $40 \%$ of the pie size, which is consistent with the literature findings.

Figures 1(a) and 1(b) summarize the acceptance and rejection rates across periods for pie size equal to HK\$136 and HK\$217, respectively.

The graphs show that the acceptance rate is more fluctuated in the first few rounds of the game. The data suggest that subjects are able to formulate strategies to stabilize acceptance rate to improve their payoffs after they learnt each other's behaviors from the market history. This is consistent with the rationale of the HCR model which assumes players respond to each other rationally, but their rationality is bounded by their knowledge about the game and how others play from the historical data.

Taking a closer look at the individual subject behavior by examining the current proposed offer $\left(p_{t}\right)$ in relation to the previous trial proposed offer $\left(p_{t-1}\right)$ of each individual subject, we find an adaptation process among subjects. Respondents always accept offers that are larger than the maximum offer in the previous round (there was only one exception that a player in Round 6 in the $\$ 136$ session rejected an offer that 


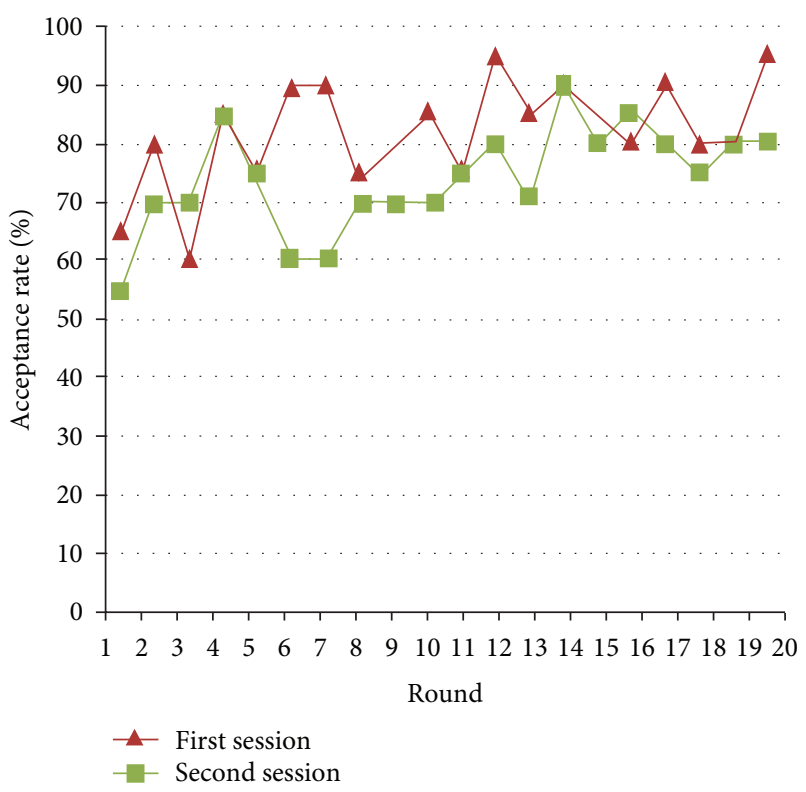

(a)

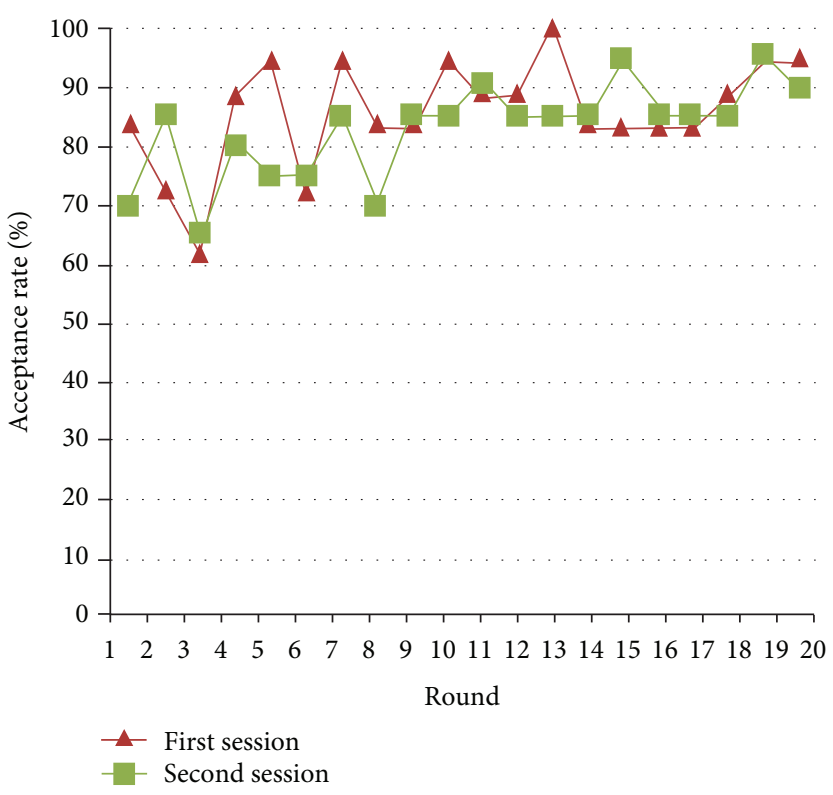

(b)

FIGURE 1: (a) Acceptance rate across rounds (pie size $=$ HK\$136). (b) Acceptance rate across rounds (pie size $=$ HK\$217).

TABLE 1: Adaptive behavior for respondents (a) (pie size $=H K \$ 136)$. (b) $($ pie size $=\mathrm{HK} \$ 217)$.

(a)

\begin{tabular}{lcc}
\hline \multirow{2}{*}{ Condition } & \multicolumn{2}{c}{ Acceptance rate } \\
& First session $(n=40)$ & Second session $(n=36)$ \\
\hline$p_{t} \geq \overline{p_{t-1}}$ & $95 \%$ & $91 \%$ \\
$p_{t}<\overline{p_{t-1}}$ & $69 \%$ & $72 \%$ \\
\hline
\end{tabular}

(b)

\begin{tabular}{lcc}
\hline \multirow{2}{*}{ Condition } & \multicolumn{2}{c}{ Acceptance rate } \\
& First session $(n=36)$ & Second session $(n=40)$ \\
\hline$p_{t} \geq \overline{p_{t-1}}$ & $94 \%$ & $94 \%$ \\
$p_{t}<\overline{p_{t-1}}$ & $74 \%$ & $59 \%$ \\
\hline
\end{tabular}

$p_{t}$ stands for the proposed offer in period $t$.

$\overline{p_{t-1}}$ stands for the mean offer in the previous period.

is $\$ 1$ higher than the maximum offer in the previous round) and accept more than $90 \%$ of the offers 1 that are larger than the mean offer in the previous round, but reject more than a quarter of the offers that are less than the mean offer in the previous round. Details are summarized in Table 1. For proposers, as shown in Table 2, there are no specific trends if their offers in the previous round were accepted. However, if proposers' offers were rejected in the previous round, most of them would propose a substantially higher offer, with a mean difference of 5.74 and 4.19 ( $P$ value of $t$-test $=0.00$ and $0.00)$ for the first and second sessions when pie size equals to HK\$136, and a mean difference of 10.44 and 4.32 ( $P$ value of $t$-test $=0.00$ and 0.00$)$ for the first and second sessions when pie size equals to $\$ 217$, respectively.
Intuitively, since proposers prefer more than less and are very afraid of being rejected and earning nothing, they would adjust their offers based on historical results. The foundation of HCR model is built based on this kind of behaviors, as the model assumes that players respond to each other by playing strategies that are derived optimally from the historical data.

There is also a rising trend of the average offers made by the proposers to the respondents across periods, which could be an indication of the learning effect among subjects. Again, HCR model can capture this. The rising trend can best be understood by studying the time series plots in Figures 2(a) and 2(b).

From the time series plots, it is evident that the offers are positively correlated with the period number for all sessions. As shown in the trend lines and the larger value of the $R^{2}$ of the regression equation, the ultimatum experiment of \$217 pie size seems to exhibit a stronger learning effect, probably due to a larger pie size that gives the proposers a larger room for profits at the beginning of the game, when the respondents have no knowledge on the actual pie size.

One of the logical explanations for the change of offers across periods is that subjects were using market information (historical data) to determine their strategies. HCR model essentially captures this kind of human behavior and decision making process. In the next two sections, we compare the two different rationalities under the HCR model, herd behavior and fictitious play, which both suggest people use historical information to assist them in making decisions.

4.2. Herd Behavior. A regression analysis shows the absolute differences between the herd behavior predicted offers and the actual offers made by subjects are decreasing across periods for both pie sizes, which suggests that herd behavior 
TABLE 2: Adaptive behavior for proposers (a) (pie size $=H K \$ 136)$. (b) (pie size $=H K \$ 217)$.

(a)

\begin{tabular}{lccccc}
\hline \multicolumn{2}{c}{$\begin{array}{c}\text { Offer was accepted in the previous round } \\
\text { Percentage }\end{array}$} & \multicolumn{2}{c}{ Offer was rejected in the previous round } \\
Current offer & First session & Second session & & \multicolumn{2}{c}{$\begin{array}{c}\text { Percentage } \\
\text { Second session }\end{array}$} \\
\hline$p_{t}>p_{t-1}$ & $22 \%$ & $21 \%$ & $p_{t}>p_{t-1}$ & $72 \%$ & $60 \%$ \\
$p_{t}=p_{t-1}$ & $53 \%$ & $57 \%$ & $p_{t}=p_{t-1}$ & $25 \%$ & $32 \%$ \\
$p_{t}<p_{t-1}$ & $24 \%$ & $22 \%$ & $p_{t}<p_{t-1}$ & $3 \%$ & $8 \%$ \\
\hline
\end{tabular}

(b)

Offer was accepted in the previous round Current offer Percentage

First session

\begin{tabular}{|c|c|c|}
\hline$p_{t}>p_{t-1}$ & $31 \%$ & $25 \%$ \\
\hline$p_{t}=p_{t-1}$ & $44 \%$ & $46 \%$ \\
\hline$p_{t}<p_{t-1}$ & $25 \%$ & $29 \%$ \\
\hline
\end{tabular}

$p_{t}$ stands for the proposed offer in period $t$.

First session

Time series plot of all, accepted and rejected offers
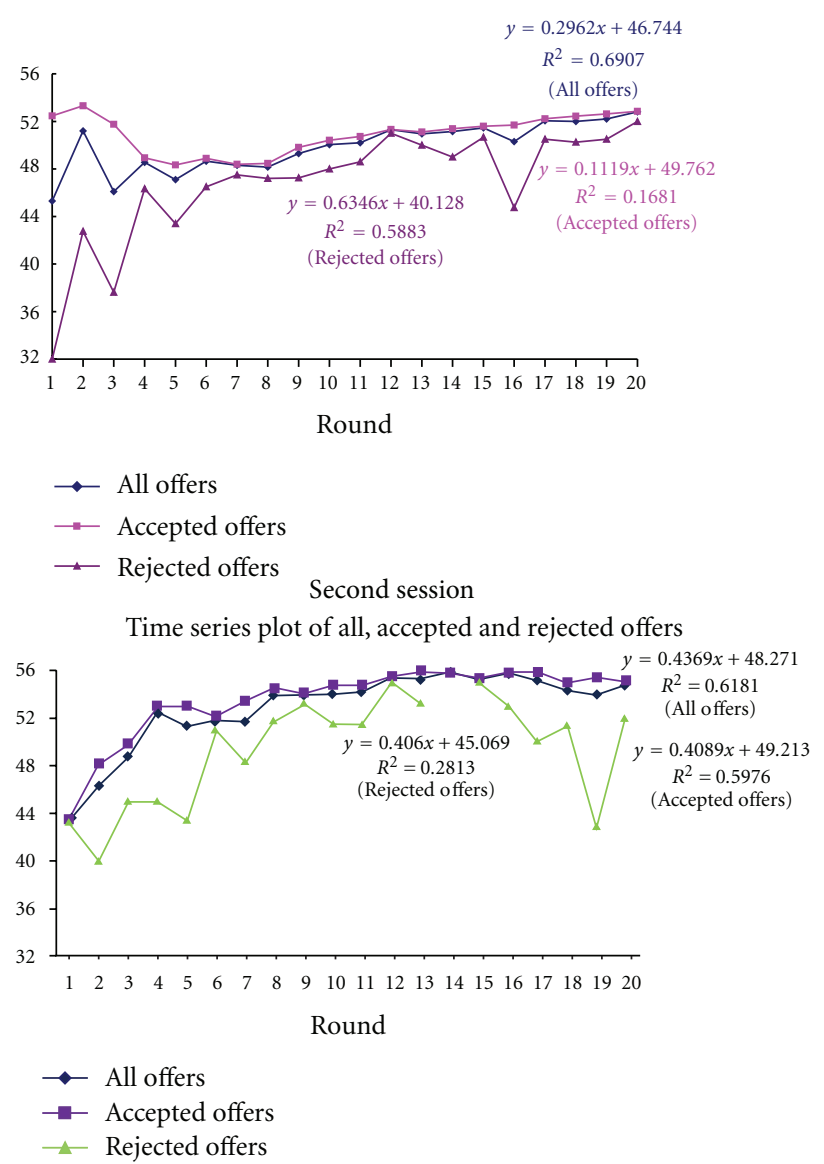

(a)

\section{First session}

Time series plot of all, accepted and rejected offers

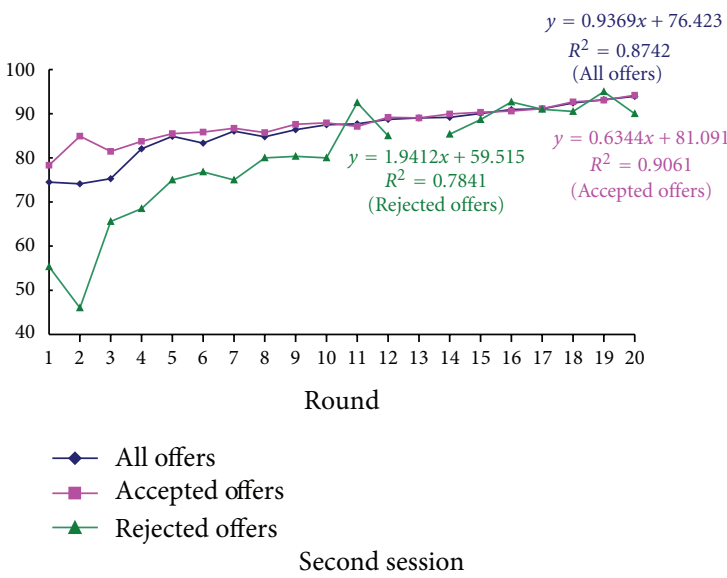

Time series plot of all, accepted and rejected offers

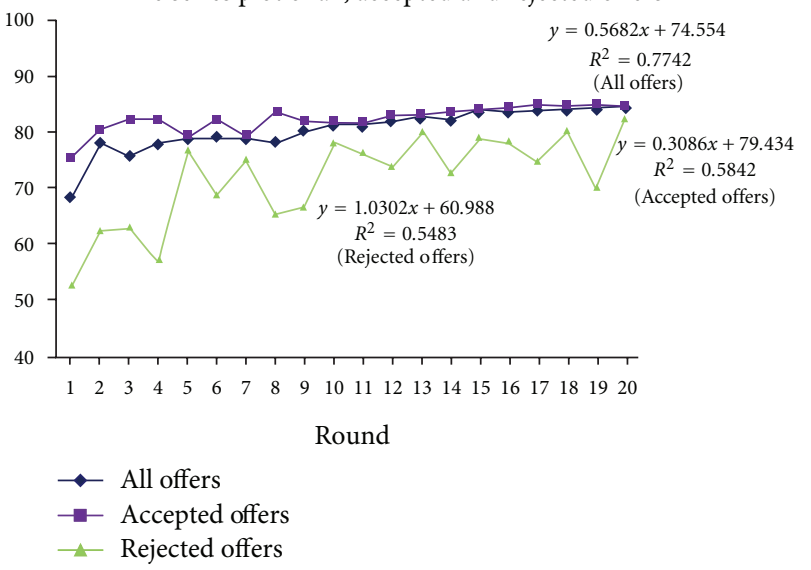

(b)

Figure 2: (a) Time series plot of offers (pie size $=$ HK\$136). (b) Time series plot of offers (pie size $=$ HK\$217). 
prediction is closer to the actual behaviors when subjects gain more experience and get more information from the historical transactions. For the $\$ 136$ pie size, the regression equation is $y=-0.386 x+7.062$, and $y=-0.360 x+8.073$, with $R^{2}$ equals to 0.177 and 0.187 , and the model is significant with $P$ value equals to 0.00 and 0.00 for the first and second sessions, respectively. For the $\$ 217$ pie size, the regression equation is $y=-0.632 x+12.966$ and $y=-0.563 x+11.920$, with $R^{2}$ equals to 0.231 and 0.147 , and the model is significant with $P$ value equals to 0.00 and 0.00 for the first and second sessions, respectively. For both equations, $y$ refers to the absolute difference between the herd behavior predicted offer and the player's actual offer, and $x$ is the period number.

On average, the absolute differences between the herd behavior prediction and the actual offers made by all subjects from the third to the final periods are $\mathrm{HK} \$ 2.63$ and $\mathrm{HK} \$ 3.93$, respectively, for the first and second sessions when pie size equals $\$ 136, \mathrm{HK} \$ 5.70$ and $\mathrm{HK} \$ 5.44$, respectively, for the first and second sessions when pie size equals HK\$217. For the detailed results of the absolute difference between herd behavior prediction and the actual offers made by each subject in each period, see Supplementary Material (Appendix 4).

4.3. Fictitious Play. A regression analysis shows the absolute differences between the fictitious play predicted offers and the actual offers made by subjects are decreasing across periods for both pie sizes, which suggests that fictitious play prediction is closer to the actual behaviors when subjects gain more experience and get more information from the historical transactions. The decreasing trend is statistically significant at 5\% level in all of the 4 sessions. For the $\$ 136$ pie size, the regression equation is $y=-0.458 x+9.203$ and $y=-0.141 x+5.830$, with $R^{2}$ equals to 0.284 and 0.034 , and the model is significant with $P$ value equals to 0.00 and 0.04 for the first and second sessions, respectively. For the \$217 pie size, the regression equation is $y=-0.528 x+10.038$ and $y=-0.580 x+13.079$, with $R^{2}$ equals to 0.153 and 0.163 , and the model is significant with $P$ value equals to 0.00 and 0.00 , respectively, for the first and second sessions. For these regression equations, $y$ refers to the absolute difference between the fictitious play predicted offer and the player's actual offer, and $x$ is the period number.

The overall mean absolute differences between the fictitious play predicted offers and the actual offers of all players is $\mathrm{HK} \$ 3.83$ and $\mathrm{HK} \$ 3.84$ for the first and second sessions, respectively, when the pie size is $\$ 136$ and $\mathrm{HK} \$ 5.53$ and HK\$6.52 for the first and second sessions, respectively, when the pie size is $H K \$ 217$. These differences are no more than $3 \%$ of the total pie size in all sessions. For the detailed results of the absolute difference between fictitious play prediction and the actual offers made by each subject in each period, see Supplementary Material (Appendix 5).

\section{Conclusions}

In this paper, historical market information is added to the ultimatum game under asymmetric information condition to study how people use market information when perfect information does not exist. A predictive model, the historyconsistent rationality model (HCR model), is introduced to explain and predict the subject behaviors. The experiment data supported the rationale of HCR model under different market conditions and showed that there was only a minimal difference between the HCR predicted behaviors and the actual behaviors.

Herd behavior and fictitious play rationalities both predict the subject behaviors reasonably well, which suggests that people tend to deploy the successful strategy used by others to be their own strategy. Though there is no conclusive evidence on whether the herd behavior or fictitious play rationalities better predict subject behaviors, for instance, when we conducted a paired samples $t$-test about the difference of the two means: (1) the absolute difference between herd behavior predicted offers and actual offers, and (2) the absolute difference between fictitious play, predicted offers and actual offers, we found that out of 20 subjects in the second session when pie size equals $\mathrm{HK} \$ 217,3$ subjects had herd behavior exhibited statistically significantly better prediction than fictitious play, while 2 subjects had fictitious play exhibited statistically significant better prediction than herd behavior, and all the remaining 15 subjects showed statistically insignificant difference at 5\% significance level. The other three sessions also have similar inconclusive findings.

On the other hand, there is strong statistical evidence that subjects best response to the empirical distribution of play in all sessions, as both herd behavior and fictitious play predictions were very close to the actual offers, especially in the later periods, which is consistent with the rationale of HCR model that suggests players respond to each other "rationally," but their rationality is bounded by their knowledge about the game and how others play, and their knowledge is derived from historical data. In other words, players behave rationally given the information learned from history.

\section{References}

[1] G. E. Bolton, E. Katok, and K. Zwick, "Dictator game giving: rules of fairness versus acts of kindness," International Journal of Game Theory, vol. 27, no. 2, pp. 269-299, 1998.

[2] E. Fehr and K. M. Schmidt, "A theory of fairness, competition, and cooperation," Quarterly Journal of Economics, vol. 114, no. 3, pp. 817-868, 1999.

[3] M. Mitzkewitz and R. Nagel, "Experimental results on ultimatum games with incomplete information," International Journal of Game Theory, vol. 22, no. 2, pp. 171-198, 1993.

[4] R. T. A. Croson, "Information in ultimatum games: an experimental study," Journal of Economic Behavior and Organization, vol. 30, no. 2, pp. 197-212, 1996.

[5] J. H. Kagel, C. Kim, and D. Moser, "Fairness in ultimatum games with asymmetric information and asymmetric payoffs," Games and Economic Behavior, vol. 13, no. 1, pp. 100-110, 1996.

[6] H. Raiffa, The Art \& Science of Negotiation, Harvard University Press, Cambridge, Mass, USA, 1982.

[7] C. C. Lee and M. J. Ferguson, "To reveal or not to reveal? Strategic disclosure of private information in negotiation," European Journal of Operational Research, vol. 207, no. 1, pp. 380-390, 2010. 
[8] J. Stahl, Bargaining Theory, Stockholm, Sweden, 1972.

[9] W. Güth, R. Schmittberger, and B. Schwarze, "An experimental analysis of ultimatum bargaining," Journal of Economic Behavior and Organization, vol. 3, no. 4, pp. 367-388, 1982.

[10] W. Güth and R. Tietz, "Ultimatum bargaining behavior. A survey and comparison of experimental results," Journal of Economic Psychology, vol. 11, no. 3, pp. 417-449, 1990.

[11] C. Camerer and R. H. Thaler, "Anomalies: ultimatums, dictators and manners," Journal of Economic Perspectives, vol. 9, no. 2, pp. 209-219, 1995.

[12] J. Ochs and A. E. Roth, "An experimental study of sequential bargaining," American Economic Review, vol. 79, no. 3, pp. 355$384,1989$.

[13] R. H. Thaler, "Anomalies: the ultimatum game," Journal of Economic Perspectives, vol. 2, no. 4, pp. 195-206, 1988.

[14] G. E. Bolton, "A comparative model of bargaining: theory and evidence," American Economic Review, vol. 81, pp. 1096-1136, 1991.

[15] S. T. Trautmann, "A Fehr-Schmidt model for process fairness," Working Paper, CREED, University of Amsterdam, 2006.

[16] W. Güth, "On ultimatum bargaining experiments-a personal review," Journal of Economic Behavior and Organization, vol. 27, no. 3, pp. 329-344, 1995.

[17] C. F. Camerer, T. H. Ho, and J. K. Chong, "A cognitive hierarchy model of games," Quarterly Journal of Economics, vol. 119, no. 3, pp. 861-898, 2004.

[18] A. V. Banerjee, "A simple model of herd behavior," Quarterly Journal of Economics, vol. 107, no. 3, pp. 797-817, 1992.

[19] G. W. Brown, "Iterative solutions of games by fictitious play," in Activity Analysis of Production and Allocation, T. C. Koopmans, Ed., John Wiley \& Sons, New York, NY, USA, 1951. 

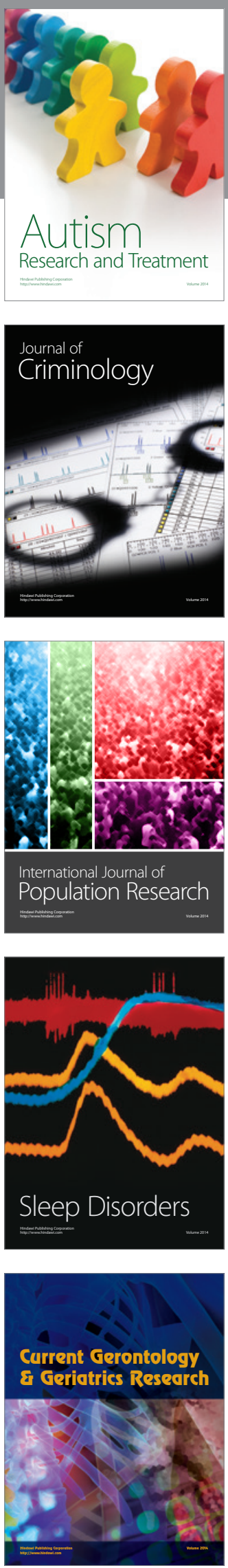
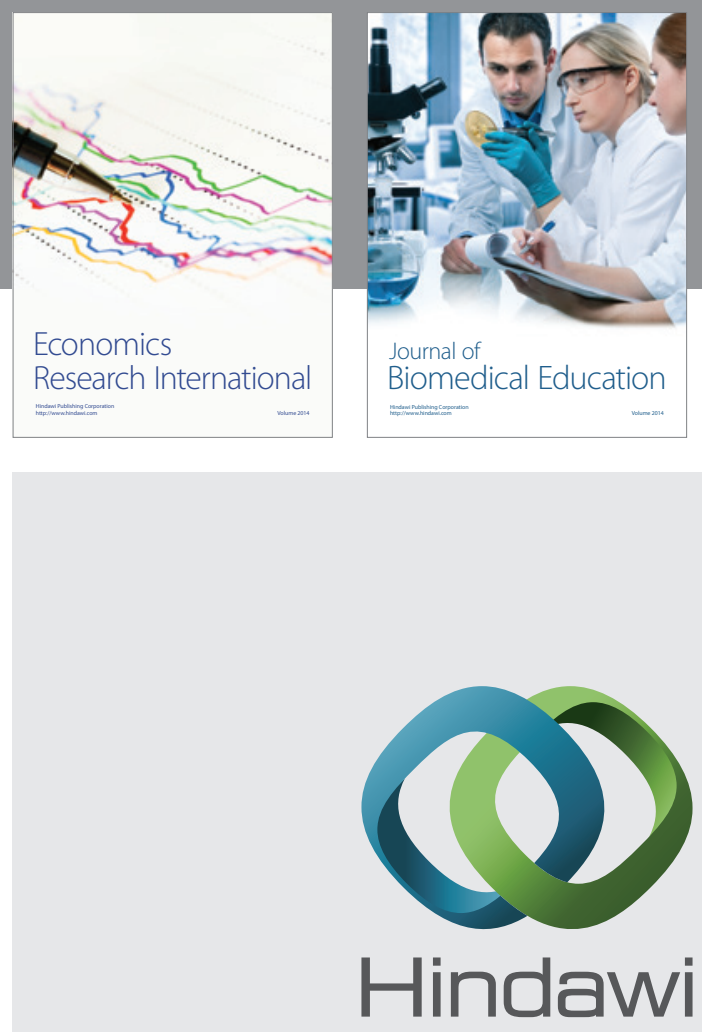

Submit your manuscripts at

http://www.hindawi.com
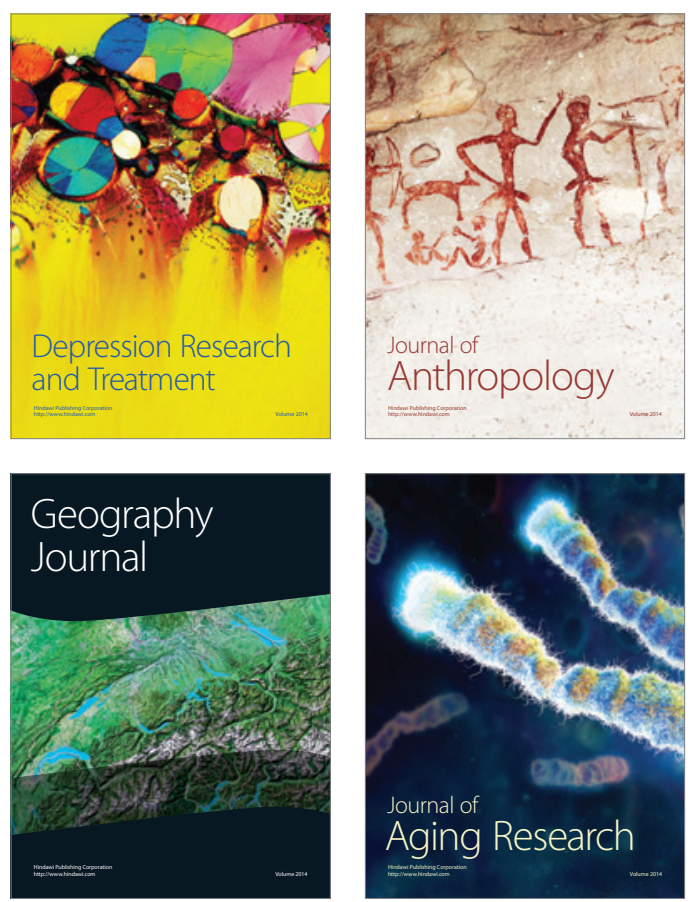
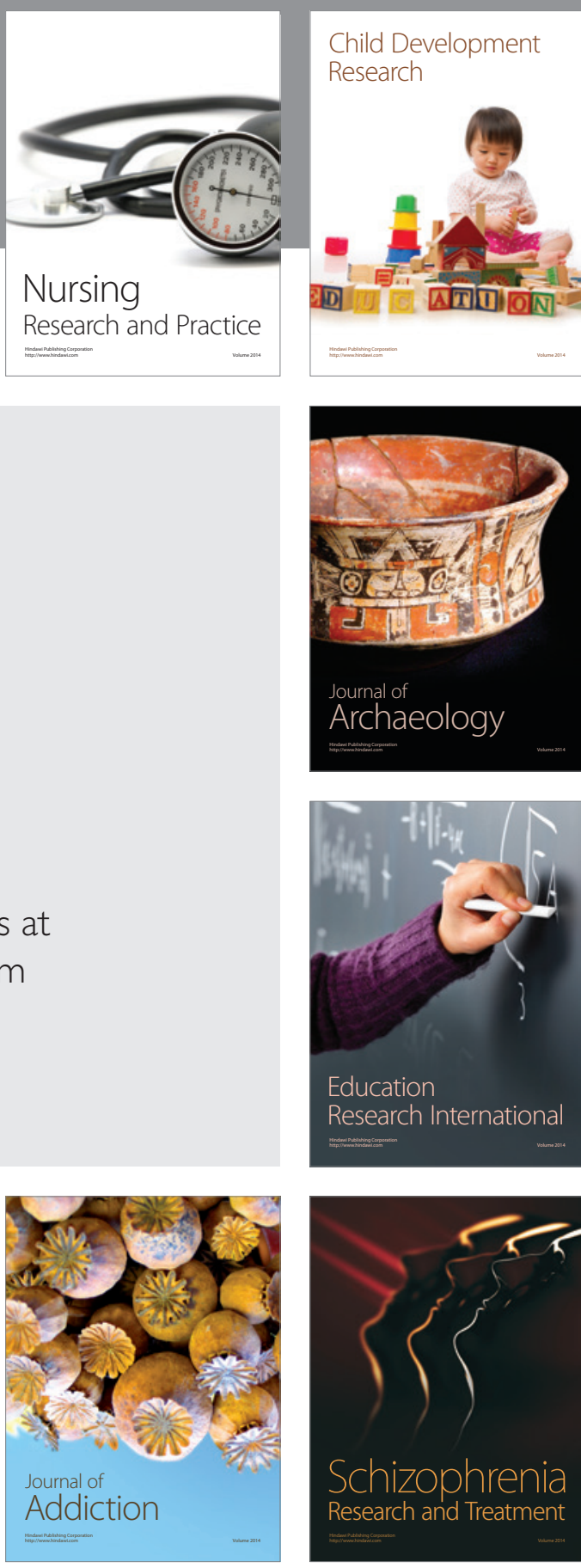

(D)
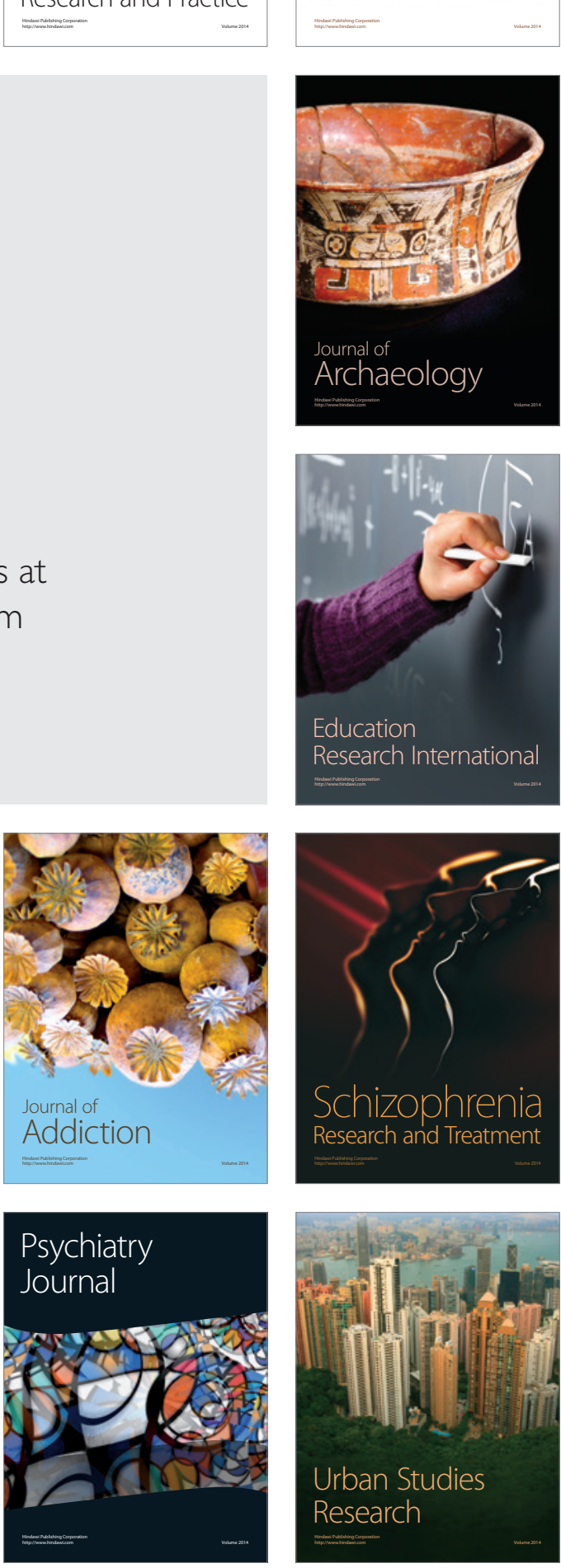